\title{
SOLVING MULTI-ROBOT PICKING PROBLEM IN WAREHOUSES: A SIMULATION APPROACH
}

\author{
Jiang, $\mathrm{H}$. \\ Shenzhen Polytechnic, Liuxian Avenue, Shenzhen, 518055, China \\ E-Mail: jianghong@ szpt.edu.cn
}

\begin{abstract}
This paper focuses on the order batching problem, aiming at this order batching problem of an ecommerce unmanned warehouse multi-robot picking system, considering the complexity and uncertainty of the system. In this paper establishes a two-stage model with the objective function of maximizing the sum of the average similarity of each picking station and balancing the picking station picking times, and uses a dynamic clustering algorithm to solve the model. The simulation results show that a two-stage order batching model that considers the order similarity and the picking time balance can be established, which can reduce the number of shelves effectively and improve the picking efficiency of warehouse multi-robot system.

(Received in August 2020, accepted in November 2020. This paper was with the author 1 month for 2 revisions.)
\end{abstract}

Key Words: Multi-Robot, Picking System, Warehouses, Two-Stage Order Batch Model; Dynamic Clustering Algorithm

\section{INTRODUCTION}

In recent years, in the research on robot systems, robots have been applied in multiple fields. Compared to single robots, multi-robot systems can cooperate and complete tasks that cannot be completed by a single robot and can ensure that the tasks are efficient and accurate. Due to the unique advantages of a multi-robot system, these systems have been attracted by many experts and scholars at home and abroad. Regarding the logistics aspect, an intelligent warehouse picking system composed of multi-robot systems has become the research focus. On the one hand, multi-robot systems can complete the picking using collaboration operations to enhance the efficiency of the order picking. On the other hand, the collaborative operating mode of multiple robots also increases the complexity of the research on these systems. In the multirobot intelligent storage picking system research, there are many studies on intelligent storage picking systems, such as warehouse planning, road network layout, storage location optimization, order batching, task scheduling, and route optimization. E-commerce orders have characteristics such as small batches, multiple varieties, and high frequency, and the orders usually must be pre-processed in the system. Pre-processing is the processing before the order picking. It is done according to certain rules, and it places orders with the same characteristics together. Picking together can reduces the amount of shelves and improves the operation's efficiency. In e-commerce warehouses, the quantity of orders is large, the variety of goods is large, and the order batching problem has proven as a Non-Deterministic Polynomial Problem. Reasonable order batching can greatly reduce the quantity of shelves, the number of transferring times and running costs of a logistics robot, thereby improving the picking system's efficiency. Order batching is one of the most basic steps of order picking operations, and a reasonable order batching strategy can effectively reduce the picking time and can allocate operations for subsequent tasks, thereby providing good initial data. This article researches the order batching and task allocation problems of an e-commerce unmanned warehouse multi-robot picking system.

The research on the order batching problem is divided into the "person-to-stock" picking operating modes and the "goods-to-person" picking operating modes. Of the two, the order 
batching problem in the "person-to-stock" picking operating mode is studied more. Henn and Wäscher [1] studied the order batching problem at a fixed time. Based on the correlation of the goods, a mathematical model with the shortest total picking time is established, and the two improved Tabu search algorithms can be used to solve the dynamic order batching problem. Zivanic et al. [2] developed an original bound cavities method, which defines the sequence of execution of received orders in "pick and pass" systems, where the tendency is to perform zone changes in places where there is a continuity of no items for extraction. Hsieh and Huang [3] adopted $K$-means clustering and self-organizing neural network classification methods (KMB and SOMB) for order batching. The experiments have proved that the methods can effectively reduce the total running time and can find the best order batch combination for different order types. Henn and Schmid [4] using a meta-heuristic algorithm minimized the delay time of orders and adopted an iterative local search method, which has been combined with the mountain climbing algorithm to solve the order sequencing problem. Klodawski et al. [5] presents analysis of the order picking system efficiency under congestion situations. Wang et al. [6] also established a two-objective order batch mathematical model that considers the total service time and balances the workload of each partition and used genetic algorithms to solve the dual-objective model. Matthews and Visagie [7] pointed out that in a "goods picking" system, order ordering has an important effect on the picking efficiency. The concept of cutting is introduced and the optimal solution is found. An order batch models are established to short the walking distance of pickers, and a heuristic saving algorithm is used to solve the model.

Regarding as the "goods-to-people" picking operation mode, Li et al. [8] addresses the problem of optimal movable-shelf selection for the cargo-to-person picking mode. However, the work does not take into account the characteristics of parallel operations of multiple picking stations. In addition, the heuristic algorithm is used to solve the order batching model. Koch and Wäscher [9] solved the order batching problem through a hybrid algorithm combining a group genetic algorithm with a local search process. There are other certain order batch processing methods. Xiang et al. [10] studied the order batching problem under a Kiva system, established a mathematical model to minimize the quantity of shelf visits, and proposed a heuristic algorithm to maximize the number of orders by improving the variable neighbourhood search. However, most of the research on order batching problems only considers defining the correlation between orders, which would reduce the number of shelves, and does not consider the picking time using a balanced picking table to improve the system's picking efficiency [11]. In an e-commerce unmanned warehouse multi-robot picking system, the picking efficiency of the system is determined by the picking table with the longest picking time [12]. Therefore, for improving the picking efficiency of the warehouse and solving the order batching problem, it is often necessary to pre-process an order to improve the picking efficiency of picking and customer satisfaction to the order.

In summary, the article researches the order to solve the batching problem of an e-commerce unmanned warehouse multi-robot picking system. It considers placing orders with a large order similarity in the same batch for picking to reduce the rack handling times and considers balanced picking table picking to improve the system's picking efficiency. Based on this, a twostage model is established in two aspects, and a dynamic clustering algorithm is designed to solve the model to achieve a reasonable order batching result. In this paper, the order allocation problem is first defined. A detail description of the order batch problem is given, the order similarity is defined by the number of times that any two orders move the same shelf, and a two-stage order batch model is established for the order correlation and picking time balance. In an order batch model, the first stage maximizes the average similarity between each picking station and reduces the number of shelves. The second stage minimizes the picking time of the largest picking station and improves the system's picking efficiency. Finally, according to the characteristics of the model, a dynamic clustering algorithm can be used to solve the models 
and complete the order batch operations. The research results of the order batch simulation in this article are given at the beginning of Section 4. The generation of the initial data ensures a more reasonable task allocation result.

\section{PROBLEM DESCRIPTION}

For improving the picking efficiency of the warehouse, there is often necessary to pre-process an order to enhance the picking efficiency greatly and customer satisfaction to the orders [13]. This research uses the order batching problem of an e-commerce unmanned multi-robot picking system and considers the order similarity of large orders that are placed in the same batch for picking to reduce the number of times that a rack is moved, and the balanced picking table picking time can improve the system's picking efficiency. In this way, a two-stage model is established in two aspects, and a dynamic clustering algorithm is designed. The model is solved to achieve reasonable order batching results.

\subsection{Order batch method}

The order batching problem refers to a process of combining orders in accordance with certain rules in order to reduce the number of times that a logistics robot moves a rack and the costs of the system's operations. The order batching problem studied in this article is described as the follows: for a certain wave of the $N$ picking order $O_{1}, O_{2}, \ldots, O_{N}$, there are multiple kinds of goods in each order on different shelves, and we assume that each kind of good is only on one shelf. Those orders that require moving the same shelf to complete the order picking operations are divided into a batch as much as possible using a picking table for picking. In addition, we consider the picking time balance of the picking table to enhance the picking efficiency of the system and cut down the operating costs of the robot.

The order batch methods [14] are mainly divided into four types: total measurement batch, time window batch, fixed order volume batch, and smart batch methods. In total measurement batching, before the picking operation and before the orders are summarized, the products in all orders are collected together, and these products are uniformly selected, which is mainly suitable for a periodic distribution. In time window batching, in a short period of time, urgent orders are accepted and goods are quickly picked in batches, which is applicable to the order requirements of the order picking for urgent shipments. In addition, the order quantity and the number of types of items should not be too much. In fixed order quantity batching, when the number of received orders reaches a pre-set threshold, they are processed in turn on first come first served to complete the operation of picking [15]. It is mainly used as a first-come-firstserved method to achieve continuous and stable operating efficiency [16]. In smart order batching, in the order system, the orders are ordered according to certain rules such as the same picking path and strong order similarity. Orders are then processed in groups. This batch method is suitable for picking in large warehouses with a large variety of stored items. The order batch method used in this article is smart order batching, which can improve the picking efficiency of the system greatly [17]. Aiming at an e-commerce unmanned warehouse picking system assessed in this paper, a two-stage target model that maximizes the sum of the average similarity of orders at each picking station to ensure the balance of the picking station picking time is found to be effective. It reduces the number of times that a rack is moved, and it can effectively improve the system's picking efficiency.

\subsection{Dynamic clustering algorithms}

Dynamic clustering is one of the clustering algorithms and is a large sample clustering method [18]. The main feature is that it conducts rough pre-classification and then gradually adjusts the 
classification until the classes are reasonably classified. The systematic clustering method has many advantages such as low computational complexity, lower computer memory requirements, and simple methods, so it is more suitable for the cluster analysis of large samples [19].

The basic ideas of dynamic clustering method are the following: arbitrarily select the initial aggregation point or provide an initial clustering result, and according to some clustering rule, continuously adjust the results until the clustering and classification results become stable.

The process of a dynamic clustering algorithm based on order batching is as follows.

Step 1. It is preferred that the data be initialized using the clustering method.

Step 2. Determine the number of cluster centres, determine the initial of cluster centres, and perform the data's initial classification.

The different clustering centres selection will lead to the different clustering results [20], so the initial clustering centre selection will greatly affect the final clustering effect. Generally, there are several methods to select the initial clustering centre.

(1) According to the amount of clustering centres, select a group of points as an initial clustering centre randomly, perform clustering with an algorithm, compare the clustering results, and select the optimal clustering result. The corresponding clustering centre is an initial clustering centre. This can select the optimal cluster centre but is computationally intensive.

(2) Randomly select an initial clustering centre. Then, the next initial clustering centre is the farthest away the clustering centre, at the same time, the subsequent clustering centre chooses the point farthest from the already selected clustering centre. The selection method can ensure the clustering centres perfect which are not only random but also scattered.

(3) Use other clustering methods to determine the clustering centre and apply it to the algorithm of the initial clustering centre.

Step 3. Regarding the clustering rules, continuously adjust the clustering centre of each cluster until the clustering centre does not change.

\section{MODELLING PROCESS}

The other order batching problem in the article is waving picking. For the orders within a certain wave, order batch processing is performed. The number of wave-picking orders is determined by the sum of the maximum number of buffers in each picking station.

\subsection{Two-stage model assumptions}

Aiming at this order batching problem of an e-commerce unmanned warehouse multi-robot picking system, considering the complexity and uncertainty of the system, the following assumptions are made:

1)Each order has at least only one kind of good, and the one kind of good can be fit in many different orders.

2) The goods of each order are indivisible.

3) The warehouse layout, order information, storage location information, shelf positions, etc. are known conditions.

4) A good is located on one shelf only.

5)Do not consider the situation that a good is out of stock.

6) The time for the staff to pick any item is the same.

7) The rated speed of the logistics robot during operations always remains the same.

8) A robot moves only one shelf at a time when serving a picking table. 


\subsection{Two-stage model construction}

\section{Parameter definition at the first stage}

The parameters and variables used in the first stage model are as follows: $n$-orders, where $n=1,2, \ldots, N ; q$ - shelves, where $q=1,2, \ldots, Q ; s$ - picking tables, where $s=1,2, \ldots, k ; W$ - the maximum capacity of the picking station when processing orders (the specific value of orders is divided by the number of picking stations multiplied by $150 \%$, that is, $W=(N / k) \times 150 \%)$;

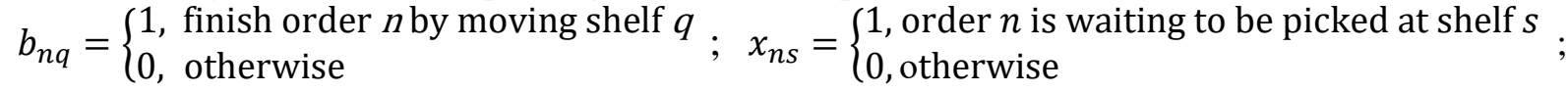
$\gamma_{n m}-$ order $m$ with similarity $n$.

Upon completion of two orders $n$ with $m$, the number of same shelves to be moved is in the range $[0 \sim 1]$. There are multiple kinds of goods on each order, different goods are on different shelves, and multiple orders need to be moved to complete an order. As much as possible, the orders are placed on the same picking table as a batch to reduce the number of times the shelves are moved.

$$
\begin{aligned}
& \gamma_{n m}=\left\{\begin{array}{cc}
\frac{\sum_{q=1}^{Q} b_{n q} b_{m q}}{\sum_{q=1}^{Q} b_{n q}+\sum_{q=1}^{Q} b_{m q}-\sum_{q=1}^{Q} b_{n q} b_{m q}} & n \neq m \\
0 & n=m
\end{array}\right. \\
& \text { The order similarity matrix } \gamma \text { is defined as follows: } \gamma=\left[\begin{array}{cccc}
\gamma_{11} & \gamma_{12} & \cdots & \gamma_{1 N} \\
\gamma_{21} & \gamma_{22} & \cdots & \gamma_{2 N} \\
\vdots & \vdots & \ddots & \vdots \\
\gamma_{N 1} & \gamma_{N 2} & \cdots & \gamma_{N N}
\end{array}\right] \text {. }
\end{aligned}
$$

There is a small example used to illustrate the correlation between orders. Suppose there are 6 orders, which are represented by order number $=$ (man who needs to be carried out to complete the order by number), that is:

$$
1=(2,3), 2=(3,9,10\}, 3=\{1,4,7\}, 4=\{1,7\}, 5=\{4,8,9\} \text {, and } 6=\{1,2,22\} .
$$

For example, $\gamma_{12}=\frac{1}{2+3-1}=0.25$. Therefore, the similarity between the 6 orders is as follows:

$$
\gamma=\left[\begin{array}{llllll}
0 & 0.25 & 0 & 0 & 0 & 0.25 \\
0.25 & 0 & 0 & 0 & 0 & 0 \\
0 & 0 & 0 & 0.67 & 0.2 & 0.2 \\
0 & 0 & 0.67 & 0 & 0 & 0.25 \\
0 & 0 & 0.2 & 0 & 0 & 0 \\
0.25 & 0 & 0.2 & 0.25 & 0 & 0
\end{array}\right]
$$

The objective function is to reduce the quantity of times that a rack is moved and to maximize the sum of the average similarity between every picking station:

$$
\operatorname{Max} Z=\sum_{s=1}^{k}\left(\frac{\sum_{n=1}^{N} \sum_{m=1}^{N} \gamma_{n m} x_{n s} x_{m s}}{\left(\sum_{n=1}^{N} x_{n s}\right)\left(\sum_{n=1}^{N} x_{n s}-1\right)}\right)
$$

Among them, the constraints are as follows:

$$
\sum_{s=1}^{k} x_{n s}=1 \quad n=1,2, \ldots, N
$$




$$
\begin{gathered}
\sum_{q=1}^{Q} b_{n q} \geq 1 \quad n=1,2, \ldots, N \\
2 \leq \sum_{n=1}^{N} x_{n s} \leq W \quad s=1,2, \ldots, k \\
x_{n s} \in\{0,1\} \quad n=1,2, \ldots, N ; s=1,2, \ldots, k \\
b_{n q} \in\{0,1\} \quad n=1,2, \ldots, N ; q=1,2, \ldots, Q
\end{gathered}
$$

Constraint (3) shows that one order can just be batched to one picking station for picking; constraint (4) indicates that at least one shelf is used to fulfil an order; constraint (5) shows that the quantity of orders on every picking station is not over $W$ and is not less than 2; and constraints (6) and (7) are variable value constraints.

\section{Parameter definition at the second stage}

Based on the first phase, the parameters and variables used in the second stage model are as follows: $j$-goods, where $j=1,2, \ldots, l ; d_{q s}$ - distance from shelf $q$ to picking table $s ; t_{p i c k}-$ the time when the staff picks a good; $v$-running speed of the robot;

$\alpha_{j n}=\left\{\begin{array}{l}1, \text { goods } j \text { is on order list } n \\ 0, \text { otherwise }\end{array} \beta_{j q}=\left\{\begin{array}{l}1, \text { goods } j \text { is on shelf } q \\ 0, \text { otherwise }\end{array} ; \rho_{q s}=\left\{\begin{array}{l}1, \text { shelf } q \text { is used by picking table } s \\ 0, \text { otherwise }\end{array}\right.\right.\right.$.

The time for a logistics robot to complete a task mainly includes three parts: $t_{1}$ represents the time from the initial location of the logistics robot to the shelf where this task is located, $t_{2}$ represents the round-trip time between the logistics robot's handling of the shelves to the picking station point, and $t_{3}$ indicates the time when the staff picked the goods, where $t_{j}=t_{1}+t_{2}+t_{3}$. When a robot performs a task, it often chooses to execute the task with the lowest costs. Here, we ignore the initial time of the robot to reach the shelf where the task is located, which results in the following:

$$
t_{j}=\frac{2 d_{q s}}{v}+t_{p i c k}
$$

To complete picking table $s$, the order picking time includes the time to complete all the tasks on the picking table, but there are goods on the same shelf, which decreases the time to complete picking table $s$. The order picking time is calculated as follows:

$$
t_{s}=\left(\sum_{n=1}^{N} \sum_{j=1}^{l} x_{n s} \times \alpha_{j n}\right) \times t_{j}-\sum_{q=1}^{Q} \rho_{q s} \times \frac{2 d_{q s}}{v} \times\left(\sum_{j=1}^{l} \beta_{j q}-1\right)
$$

According to the above description, the mathematical model of the following order batch problem is established:

$$
\begin{gathered}
\text { Min Max } t_{s} \\
\sum_{q=1}^{Q} \beta_{j q}=1 \quad j=1,2, \ldots, l \\
\sum_{j=1}^{l} \alpha_{j n} \geq 1 \quad n=1,2, \ldots, N \\
\sum_{q=1}^{Q} \beta_{j q} \times b_{n q} \geq \alpha_{j n} \quad j=1,2, \ldots l ; n=1,2, \ldots, N \\
\beta_{j q} \in\{0,1\} \quad j=1,2, \ldots, l ; q=1,2, \ldots, Q \\
\alpha_{j n} \in\{0,1\} \quad j=1,2, \ldots, l ; n=1,2, \ldots, N
\end{gathered}
$$

Here, objective function (10) minimizes the order picking time on the picking table with the longest picking time; constraint (11) ensures that one kind of good is only on one shelf; constraint (12) ensures that there is at least one good in an order; constraint (13) ensures that 
the goods in each order can be picked; and constraints (14) and (15) are the constraints on the values of variables.

\section{MODEL SOLUTION AND SIMULATION}

In this article, the dynamic clustering algorithms are used to resolve the model, and the main steps include the followings: the dynamic clustering algorithms first conducts rough classification, and then the classification is gradually adjusted until it is more reasonable. For the two-stage model established in this paper, in the first stage of its construction, the initial clustering results are obtained based on maximizing the similarity of each picking station, and then the clustering results are adjusted according to the picking time of the second stage to balance the picking stations. with the problems of this paper studied, a class of dynamic clustering algorithms are used to resolve the model [21]. Based on the similarity of the completed order's handling of shelves and the picking time of the balanced picking table, the model is resolved by using a dynamic clustering algorithm. The algorithm flows are shown in Fig. 1.

Input: Order information for a certain wave.

(1) A preliminary clustering process based on maximizing the sum of the average similarity of each picking station order.

Step 1. Determine the initial cluster centre.

(1) Calculate the similarity between any two orders using Eq. (1) for $\gamma_{\alpha \beta}$ to obtain the order similarity matrix $\gamma$.

(2) In the order similarity matrix, select the order that has the highest similarity with $\alpha$ other orders (the sum of the similarity between each order and other orders $\div$ number of orders). As the initial clustering centre, assign it to a picking table, and then select $\alpha$ orders with the least order correlation and the largest average similarity to the remaining orders, where $\beta$ is the initial clustering centre for the next order. Continue this process until each picking station is assigned to an initial clustering centre, where the number of initial clustering centres is based on the number of picking stations $k$ that was set.

Step 2. Order batching rules.

(1) Calculate the average similarity value of each order and each clustered order and group them according to the largest average similarity value until $k$ is one. If all the orders are included in each cluster, the process ends and the next step is performed.

(2) Determine the number of orders in each category. If the number of orders in a certain category exceeds the maximum of the picking capacity table $W$ and delete the order with the smallest average similarity with other orders in this cluster and calculate the number of orders and orders less than $W$. Based on the average similarity of the cluster, add the order to the cluster with the largest average similarity. Continue to adjust the number of orders over $W$ clusters until the total number of clusters does not exceed $W$.

(3) All orders are assigned to the corresponding picking station, and step 2 is repeated, considering the dynamic clustering adjustment of the picking time balance of each picking station.

(2) Dynamic clustering adjustment considering the picking time's balance of each picking station.

Step 3. Dynamic adjustment of the balance. In the previous step, an initial order batching result was obtained, and each order has a branch of orders.

(1) According to the initial order batching results obtained at the previous step, use Eqs. (3) to (9) to calculate the time for each picking station to complete the order picking operation. Denote the picking station with the longest picking time as $s_{1}$ and the picking station with the shortest picking time as $s_{2}$; calculate the picking time difference between the two as $t_{1}$. 
(2) For the picking station $s_{1}$, calculate the average similarity of each order on the order and find the order with the smallest average similarity $i$.

(3) Place the order $i$. Calculate the correlations with the remaining picking order cluster orders to find the cluster with the largest average similarity.

(4) After one order is added on the picking station, if the picking station's order quantity does not exceed $W, i$ more orders are added to this picking station, and we recalculate the time difference between the picking station with the longest picking time and the picking station with the shortest picking time $t_{2}$. Otherwise, we return to (3).

(5) Compare $t_{1}$ and $t_{2}$. If $t_{1}<t_{2}$, cancel the merging with this cluster. If $t_{1} \geq t_{2}$, then merge the orders with the cluster and repeat step 3 , which is the dynamic adjustment of the equilibrium, until all $t_{1}<t_{2}$. So far, the order batch operation is completed.

Output: Order number for each cluster.

\section{RESULTS AND DISCUSSIONS}

To prove the task allocation model's effectiveness and the method of the e-commerce unmanned warehouse multi-robot picking system, simulation was performed using MATLAB, and after multiple iterative calculations, the simulation results of task allocation were obtained. Taking enterprise, A, as an e-commerce company, for example, assume that the company's warehouse layout is shown in Fig. 2. In a $2000 \mathrm{~m}^{2}$ warehouse, there are 120 shelves.

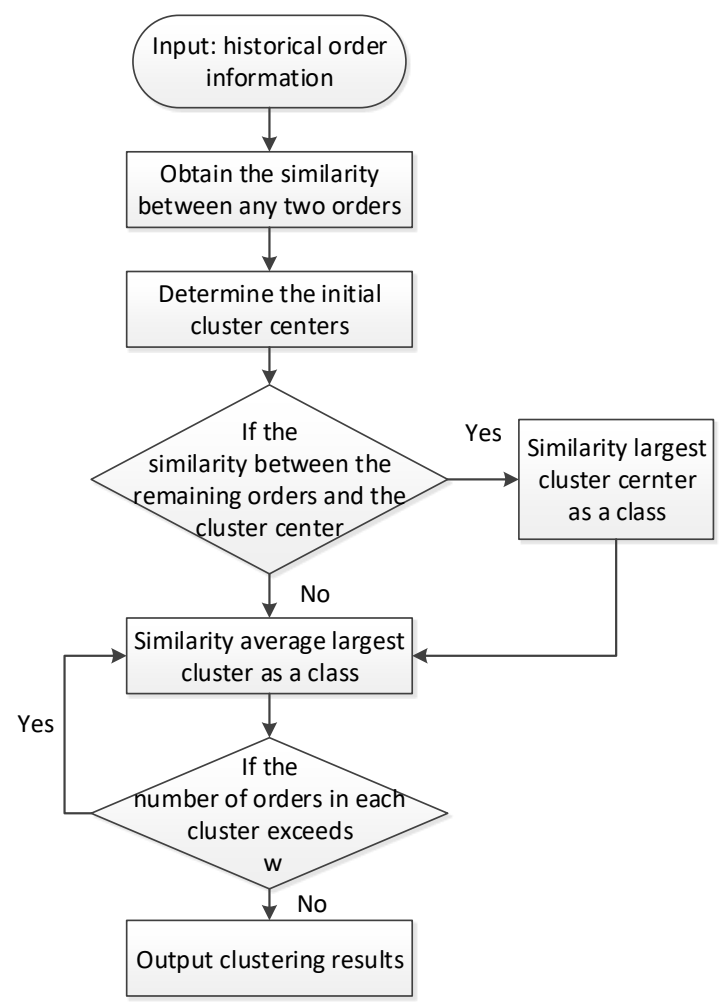

(1) The first stage

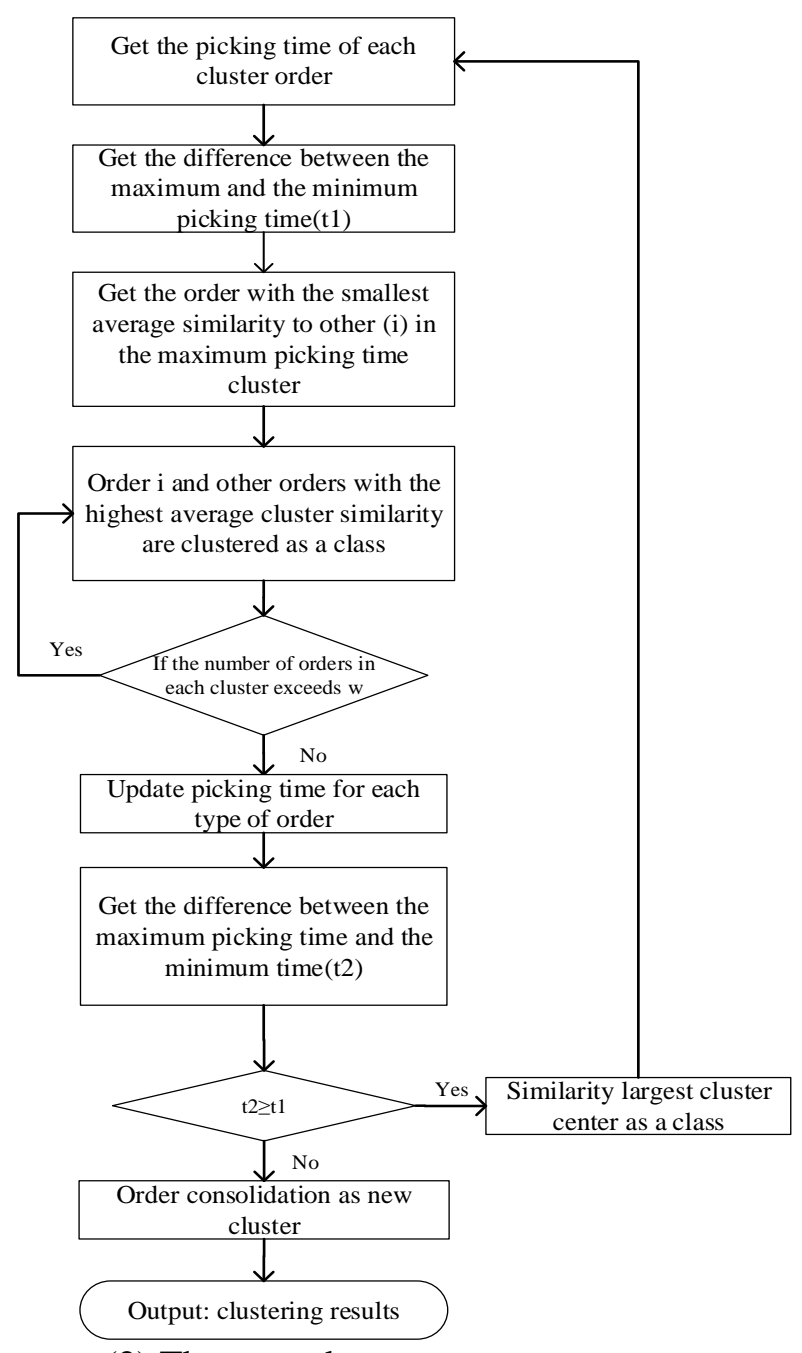

(2) The second stage

Figure 1: Algorithm design in the first stage and the second stage. 
To improve the picking efficiency, the orders under a certain wave time which are divided into batches, the orders in the batches are separated into tasks, and picking operations are assigned to the appropriate robots to perform until the picking operations of the batch order are completed [22]. The proposed model and method are verified by a simulation, and the description of the parameters of the simulation is shown in Table I.

Table I: Parameter description.

\begin{tabular}{|c|l|c|}
\hline Symbol & \multicolumn{1}{|c|}{ Description } & Value \\
\hline$R$ & Number of robots & 10 \\
\hline$T$ & Task types & 800 \\
\hline$S$ & Number of picking stations & 3 \\
\hline Order & Number of orders & 100 \\
\hline$P$ & Number of shelves & 480 \\
\hline$V$ & Robot rated speed (m/s) & 1 \\
\hline$t_{\text {pick }}$ & Staff picking speed (person/person) & 4 \\
\hline$C_{1}$ & No-load cost factor of logistics robot & {$[0 \sim 1]$} \\
\hline$C_{2}$ & Logistics robot load cost factor & {$[0 \sim 1]$} \\
\hline
\end{tabular}

To facilitate this research, orders are simulated and generated according to the characteristics of "small batch, multifrequency" of e-commerce orders. Furthermore, for increasing the data's authenticity, the products are classified according to the $\mathrm{ABC}$ classification method, in which category A products to account for $10 \%$ of the total, category $\mathrm{B}$ products to account for $20 \%$ of the total, and category $\mathrm{C}$ products to account for $70 \%$ of the total, and the corresponding product quantities are 1-50, 51-150, and 151-550, respectively. In addition, the shelves where the goods are located also correspond to category A product shelves, category B product shelves, and category $\mathrm{C}$ product shelves, accounting for $10 \%, 20 \%$, and $70 \%$ of the total, respectively, with the shelf numbers of 1-12,13-36, and 37-120. The ratio of the three types of goods $(a, b$, and $c)$ on the shelves is set as 5:3:2. There are 4-8 types of items in each order. For the simulated storage information, type a products are stored on type a shelf, type $\mathrm{b}$ products are stored on type $\mathrm{b}$ shelves, and type $\mathrm{c}$ products are stored on type $\mathrm{c}$ shelves. The random storage rules ensure that there is at least one kind of good on each shelf when obtaining the storage location information. For the 100 orders generated by a certain wave, they are processed through the abovementioned order batch process, and the number of each type of order does not exceed $W=(N / k) \times 150 \%=50$. According to the order batching model and algorithm that maximize the average similarity of each picking station and balances the picking station picking times, the order batching results are obtained, as shown in Table II.

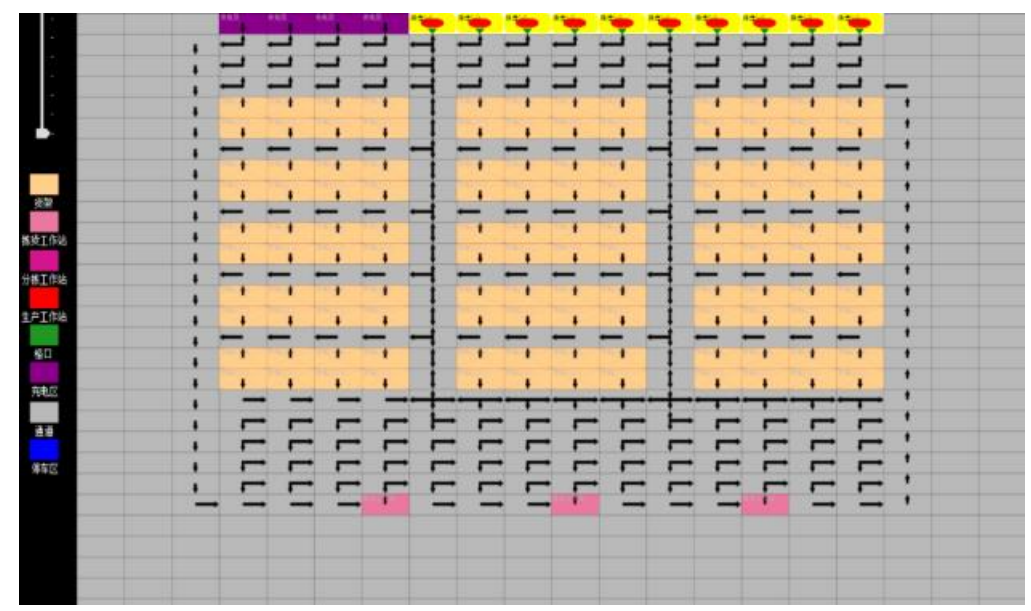

Figure 2: Warehouse layout of enterprise A. 
To prove the order batching strategy proposed is effectiveness in this article, different orders quantities were selected, which are $n=100,200,300,400,500,600,700,800,900$, and 1000. The picking station with the longest picking time determines the picking efficiency of the entire system. The lower the longest picking time is, the higher the picking efficiency. Therefore, the results of the order batching strategy of this article (considering the order correlation and picking time balance), the order batching strategy considering only the order correlation, and the random batch strategy are analysed with respect to the number of moving shelves and the picking time of the longest picking station.

(1) Contrast analysis of the number of times that a shelf is handled. Fig. $3 \mathrm{a}$ is a comparison chart of the number of times the shelves are moved in different orders for different order quantities. As the quantity of orders rises, the similarity between the orders will also increase, and the number of shelves will increase slowly. It can be seen that because of proposing the order batching strategy in this article and the order batching strategy that only considers the order similarity are less than the random order batching strategy, proposing the order batching strategy in this article can greatly reduce the quantity of shelves.

(2) Comparative analysis of the picking efficiency. Fig. $3 \mathrm{~b}$ is a comparison chart of the picking efficiency for different order batching strategies and different order quantities. As the quantity of orders rises, the picking time becomes increasingly longer. By a comparative analysis with three methods, for the order batching strategy proposed in this article, the longest picking table picking time is the smallest, which can ensure the balance of the picking table picking time, avoid congestion during the robot handling process, and effectively improve the system's picking efficiency.

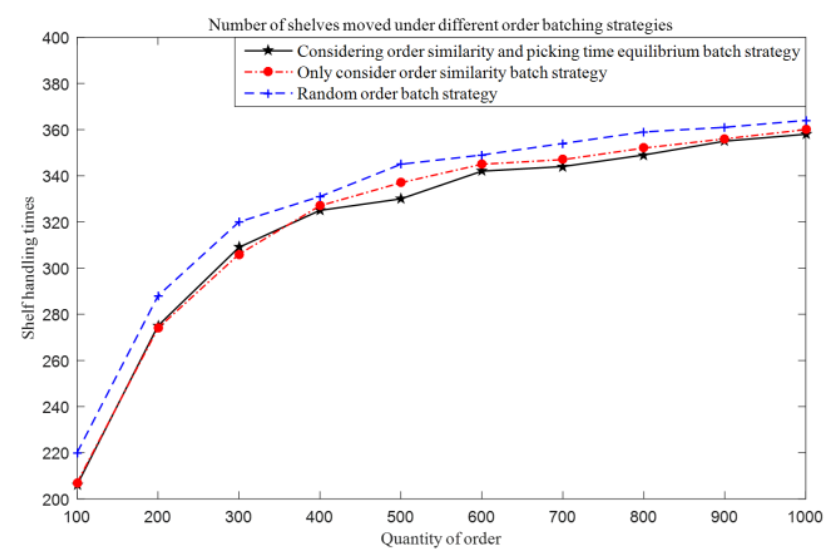

a)

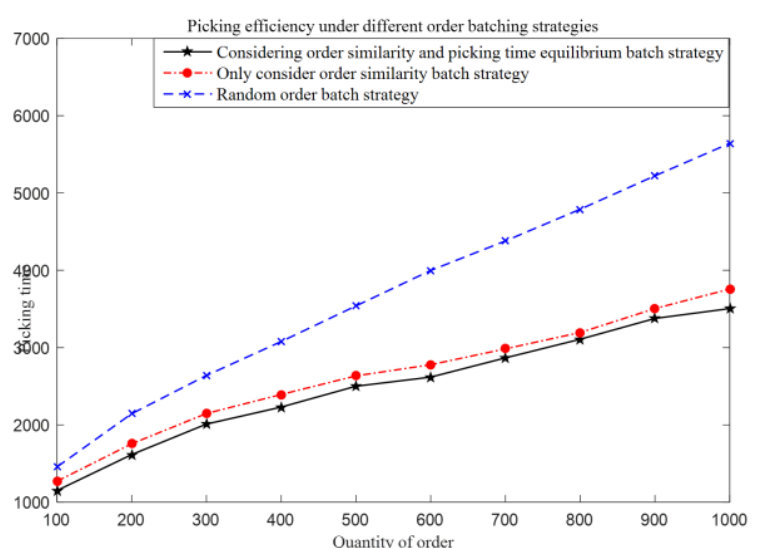

b)

Figure 3: The number of racking times and the picking time under the three order batching strategies.

\section{CONCLUSION}

This paper proposes a multi-robot task allocation model and method for an e-commerce unmanned warehouse multi-robot picking system, and simulates and verifies the warehousing picking operations of e-commerce company A. First, regarding the order batching problem, after batching different orders, the comparison of the rack handling times and the picking costs under the strategy validates the effectiveness of the order batching model and algorithm. Next, a description is provided for the order batching problem, and then a two-stage order batching mathematical model is established that considers the order correlation and the picking time balance. In the first stage, the correlation between the orders is defined by the quantity of times that any two orders are moved to the same shelf. A mathematical model that maximizes the sum of the average similarity of each picking station is established. Picking is performed using the picking table to reduce the number of times that a rack is moved. In the second stage, a 
mathematical model that minimizes the maximum picking table picking time is established according to the picking time of each picking table to enhance the system's picking efficiency. Finally, a dynamic clustering algorithm can greatly use to resolve the model.

\section{ACKNOWLEDGEMENT}

This research has been supported by Major Program of National Natural Science Foundation of China (No.:71831001). This research has been also supported by Yueqi Youth Scholar Funding of China University of Mining and Technology (Beijing).

\section{REFERENCES}

[1] Henn, S.; Wäscher, G. (2012). Tabu search heuristics for the order batching problem in manual order picking systems, European Journal of Operational Research, Vol. 222, No. 3, 484-494, doi:10.1016/j.ejor.2012.05.049

[2] Zivanic, D.; Zelic, A.; Lalic, B.; Simeunovic, N.; Szabo, L. (2019). Improving the order picking efficiency by optimising the orders' sequence, International Journal of Simulation Modelling, Vol. 18, No. 1, 125-137, doi:10.2507/IJSIMM18(1)469

[3] Hsieh, L.-F.; Huang, Y.-C. (2011). New batch construction heuristics to optimise the performance of order picking systems, International Journal of Production Economics, Vol. 131, No. 2, 618630, doi:10.1016/j.ijpe.2011.02.006

[4] Henn, S.; Schmid, V. (2013). Metaheuristics for order batching and sequencing in manual order picking systems, Computers \& Industrial Engineering, Vol. 66, No. 2, 338-351, doi:10.1016/j.cie.2013.07.003

[5] Klodawski, M.; Jachimowski, R.; Jacyna-Golda, I.; Izdebski, M. (2018). Simulation analysis of order picking efficiency with congestion situations, International Journal of Simulation Modelling, Vol. 17, No. 3, 431-443, doi:10.2507/IJSIMM17(3)438

[6] Wang, X.-P.; Zhang, W.; Yi, C.-Y. (2017). Optimizing operation efficiency of e-commerce manual synchronized zone order picking system, Journal of Industrial Engineering and Engineering Management, Vol. 31, No. 2, 209-215, doi:10.13587/j.cnki.jieem.2017.02.025

[7] Matthews, J.; Visagie, S. (2013). Order sequencing on a unidirectional cyclical picking line, European Journal of Operational Research, Vol. 231, No. 1, 79-87, doi:10.1016/ j.ejor.2013.05.011

[8] Li, Z. P.; Zhang, J. L.; Zhang, H. J.; Hua, G. W. (2017). Optimal selection of movable shelves under cargo-to-person picking mode, International Journal of Simulation Modelling, Vol. 16, No. 1, 145-156, doi:10.2507/IJSIMM16(1)CO2

[9] Koch, S.; Wäscher, G. (2016). A grouping genetic algorithm for the Order Batching Problem in distribution warehouses, Journal of Business Economics, Vol. 86, No. 1-2, 131-153, doi:10.1007/s11573-015-0789-x

[10] Xiang, X.; Liu, C.; Miao, L. (2018). Storage assignment and order batching problem in Kiva mobile fulfilment system, Engineering Optimization, Vol. 50, No. 11, 1941-1962, doi:10.1080/ $\underline{0305215 X .2017 .1419346}$

[11] Boysen, N.; Briskorn, D.; Emde, S. (2017). Parts-to-picker based order processing in a rackmoving mobile robots environment, European Journal of Operational Research, Vol. 262, No. 2, 550-562, doi:10.1016/j.ejor.2017.03.053

[12] Yu, J. (2016). Intractability of optimal multirobot path planning on planar graphs, IEEE Robotics \& Automation Letters, Vol. 1, No. 1, 33-40, doi:10.1109/LRA.2015.2503143

[13] Goyal, V.; Levi, R.; Segev, D. (2016). Near-optimal algorithms for the assortment planning problem under dynamic substitution and stochastic demand, Operations Research, Vol. 64, No. 1, 219-235, doi:10.1287/opre.2015.1450

[14] Koubaa, A.; Bennaceur, H.; Chaari, I.; Trigui, S.; Ammar, A.; Sriti, M.-F.; Alajlan, M.; Cheikhrouhou, O.; Javed, Y. (2018). Robot Path Planning and Cooperation, Springer International Publishing, Cham, doi:10.1007/978-3-319-77042-0

[15] Lee, D.-H. (2018). Resource-based task allocation for multi-robot systems, Robotics and Autonomous Systems, Vol. 103, 151-161, doi:10.1016/j.robot.2018.02.016 
[16] Klodawski, M.; Jachimowski, R.; Jacyna-Golda, I.; Izdebski, M. (2018). Simulation analysis of order picking efficiency with congestion situations, International Journal of Simulation Modelling, Vol. 17, No. 3, 431-443, doi:10.2507/IJSIMM17(3)438

[17] Nedjah, N.; Mendonca, R. M. de; Mourelle, L. de M. (2015). PSO-based distributed algorithm for dynamic task allocation in a robotic swarm, Procedia Computer Science, Vol. 51, 326-335, doi:10.1016/j.procs.2015.05.250

[18] Enright, J.; Wurman, P. R. (2011). Optimization and coordinated autonomy in mobile fulfillment systems, AAAI Conference on Automated Action Planning for Autonomous Mobile Robots, 33-38

[19] Zhou, L.; Shi, Y.; Wang, J.; Yang, P. (2014). A balanced heuristic mechanism for multirobot task allocation of intelligent warehouses, Mathematical Problems in Engineering, Vol. 2014, Paper 380480, 10 pages, doi:10.1155/2014/380480

[20] Lerher, T.; Borovinsek, M.; Ficko, M.; Palcic, I. (2017). Parametric study of throughput performance in SBS/RS based on simulation, International Journal of Simulation Modelling, Vol. 16, No. 1, 96-107, doi:10.2507/IJSIMM16(1)8.372

[21] Ongus, R. W.; Nyamboga, C. M. (2019). Collecting development practices in using information technology: a comparative study, Journal of Logistics, Informatics and Service Science, Vol. 6, No. $2,1-22$

[22] Leu, X.; Ashoka, K. (2019). Supply chain management in prefabricated construction: an overview of a developed conceptual framework, Journal of System and Management Sciences, Vol. 9, No. 2, 43-80, doi:10.33168/JSMS.2019.0203 